\title{
Anonymous Routing in Wireless Mobile Ad Hoc Networks to Prevent Location Disclosure Attacks
}

\author{
Arjan Durresi ${ }^{1}$, Vamsi Paruchuri ${ }^{1}$, Mimoza Durresi $^{2}$, and Leonard Barolli ${ }^{2}$ \\ 1 Department of Computer Science, Louisiana State University, \\ 298 Coates Hall, Baton Rouge, LA, 70803, USA \\ \{durresi, paruchuri\}@csc.lsu.edu \\ http://www.csc.1su.edu/ durresi \\ 2 Department of Information and Communication Engineering, \\ Faculty of Information Engineering, Fukuoka Institute of Technology, \\ 3-30-1 Wajiro-Higashi, Higashi-ku, Fukuoka 811-0295, Japan \\ durresim@franklin.edu, barolli@fit.ac.jp
}

\begin{abstract}
Wireless Ad Hoc networks are particularly vulnerable due to their fundamental characteristics such as an open medium, dynamic topology, distributed cooperation and constrained capability. Location information of nodes can be critical in wireless ad hoc networks, especially in those deployed for military purposes. In this paper, we present a set of protocols for anonymous routing to prevent location disclosure attacks in wireless ad hoc networks.
\end{abstract}

\section{Introduction}

Recent wireless research indicates that wireless Mobile Ad Hoc Networks (MANET) present larger security problems than conventional wired and wireless networks [1, 2]. In the traditional Internet, routers within the central parts of the network are owned by a few well-known operators and are therefore assumed to be somewhat trustworthy. This assumption no longer holds in an Ad Hoc network, since all nodes entering the network are expected to take part in routing. Also, because the links are usually wireless, any security that was gained because of the difficulty of tapping into the network is lost. Furthermore, because the topology in such a network can be highly dynamic, traditional routing protocols can no longer be used. Thus, Ad Hoc network has much harder security requirements than the traditional network and the routing in Ad Hoc networks is an especially hard task to accomplish securely, robustly and efficiently.

In general, wireless MANETs are particularly vulnerable due to their fundamental characteristics such as open medium, dynamic topology, absence of central authorities, distributed cooperation and constrained capability. The existing security solutions for wired networks cannot be applied directly in wireless MANETs.

Applications that make use of ad hoc routing have heterogeneous security requirements. Authentication, message integrity, and non-repudiation to an ad hoc environment are part of a minimal security policy. Apart from these, there 
are several other security issues [1, 3] such as black hole attacks, denial of service, and information disclosure.

A location disclosure attack can reveal something about the locations of nodes or the structure of the network. The information gained might reveal which other nodes are adjacent to the target, or the physical location of a node. In the end, the attacker knows which nodes are situated on the route to the target node. If the locations of some of the intermediary nodes are known, one can gain information about the location of the target as well.

In many cases, the location information might be very crucial. In MANETs installed for tactical/military missions in a hostile and/or unknown territory, these types of attacks have to be prevented. In many cases, the communicating nodes need to be anonymous - no other node in the network should know who is communicating with whom. The organization of the paper is as follows: In Section 2 we explain the goals of our work, in Section 3 we summarize the background work, in Section 4 we present PAR, a solution that achieves complete anonymity and discuss trade-offs between complete anonymity and difficulty in identifying misbehaving nodes, in Section 5 we present enhancements to our protocol, in Section 6 we present our conclusions.

\section{Goals}

\subsection{Anonymity}

There are three types of anonymous communication properties: sender anonymity, receiver anonymity, and unlinkability of sender and receiver [16, [17. Sender anonymity means that the identity of the party who sent a message is hidden, while its receiver (and the message itself) might not be. Receiver anonymity similarly means that the identity of the receiver is hidden. Unlinkability of sender and receiver means that although the sender and receiver can each be identified as participating in some communication, they cannot be identified as communicating with each other.

We also specify two degrees of anonymity - absolute and quasi-absolute. For simplicity, we describe these with respect to sender anonymity, but they can be extended to receiver anonymity and unlinkability as well. With absolute privacy, the attacker can in no way distinguish the situations in which a potential sender/receiver actually sent/received communication and those in which it did not. That is, sending a message results in no observable effects for the attacker. The identity and the location of each node in the network remain anonymous and sender and receiver are unlinkable. In case of quasi-absolute privacy, the neighboring nodes of the sender would be able to identify it, when it instantiates a connection and each node in the path of an established connection knows its next and previous hop neighbors' identities. Nonetheless, the receiver remains anonymous and the sender and receiver remain unlinkable unless all the nodes in the path collaborate.

It might be impossible to conceal the location itself - upon receiving a packet from a neighbor, as a node can easily approximate the location of the neighbor 
by measuring the received signal strength and angle of arrival [23]. But, here we focus on making it impossible for a node to make a correspondence between the locations and the identities of the nodes. Thus, even though a node is able to figure out that some node is present at a particular location, it would not be able to find out the identity of that node.

\subsection{What we Achieve}

Initially, we present Protocol for Anonymous Routing (PAR), based on public key cryptography, to provide absolute anonymity. With this we achieve complete sender and receiver anonymity. Also, the sender and the receiver cannot be linked to each other even if all the nodes in the established path collaborate. However, with absolute anonymity, defending against denial-of-service attacks by compromised nodes becomes very difficult. We discuss this issue in more detail in Section 4. Hence, to detect and defend against these attacks, we present PAREnhanced, a variation of the above protocol, which only provides quasi-absolute anonymity as discussed in Section 5 .

We consider the anonymity properties provided to an individual node against two distinct types of attackers:

- A local eavesdropper is an attacker who is also the neighbor of the sender/ receiver and hence, can observe all (and only) communication to and from the sender/receiver.

- Collaborating nodes are other nodes that can pool their information.

PAR guaranties sender and receiver anonymity as well as unlinkability between sender and receive against both local eavesdroppers and collaborating attackers. Compared to PAR, in PAR-E the sender or the receiver are exposed to local eavesdroppers.

\subsection{What we do not Achieve}

Our objective is to provide anonymity for the sender and the receiver. In MANETs deployed specifically for military and tactical reasons, the identity and location information of the sender and the receiver might be critical. With absolute anonymity, the identities of every node in the network are completely anonymous. But, absolute anonymity makes it difficult, if not impossible, to detect misbehaving and compromised nodes in the network. A malicious node can refuse to forward packets or may just inject unnecessary packets into the network thus resulting in denial-of-service. Even intrusion detection systems [13, [14, [15] will be of little use. For networks where absolute anonymity is not as critical as in military networks, we can trade absolute anonymity a little so as to make detection of misbehaving nodes easy.

The protocol PAR that we present for achieving absolute anonymity makes no effort to defend against denial-of-service attacks. We believe that such attacks are inherent to networks where nodes are completely anonymous. But, with PAR-E, which provides quasi-absolute anonymity, intrusion detection systems [13], [14], 15. can be used with little or no modifications to detect misbehaving nodes. 


\section{Related Work}

Ad hoc wireless networks assume no pre-deployed infrastructure for routing packets end-to-end in a network, and instead rely on intermediary peers. Securing ad hoc routing presents challenges because each user brings to the network their own mobile unit, without the centralized policy or control of a traditional network. Many ad hoc routing protocols such as such as Dynamic Source Routing (DSR), Ad Hoc On Demand Distance Vector (AODV), Zone Routing Protocol (ZRP), and Location Aided Routing (LAR) have been proposed previously, but none of the proposals have defined security requirements, and all inherently trust all participants.

All proposed protocols have security vulnerabilities and exposures that easily allow for routing attacks. These vulnerabilities are common to many protocols. The fundamental differences between ad hoc networks and standard IP networks necessitate the development of new security services. In particular, the measures proposed for IPSec [7] help only in end-to-end authentication and security between two network entities that already have routing between them; IPSec does not secure the routing protocol. While mechanisms similar to those used in IPSec can be adapted to secure the routing, IPSec alone does not suffice.

This point has been recognized, and others have started to examine security problems in ad hoc networks. A solution that uses threshold cryptography as a mechanism for providing security to the network is presented in [8]. A method that ensures equal participation among members of the ad hoc group, and that gives each node the authority to issue certificates is presented in [9]. An effort to secure an existing ad hoc routing protocol has also recently been made available [10].

Apart from the above protocols, which try to deal with minimal security requirements like Authentication, message integrity, and non-repudiation, several other protocols were presented to deal with specific security issues encountered in MANETs. 4] presents the resurrecting duckling security policy model, which describes secure transient association of a device with multiple serialized owners. [5] presents a solution to prevent black hole attacks, 6] presents strategies for intrusion detection.

Anonymous communication for wired networks is a well-studied aspect. The concept of a mix is introduced in [18. A single processor in the network, called a mix, serves as a relay. Each processor $\mathrm{P}$ that wants to send a message $\mathrm{m}$ to a processor Q encrypts $\mathrm{m}$ using Q's public key to obtain $\mathrm{m}$ '. Then $\mathrm{P}$ encrypts the pair (m', q) using the public key of the mix. The mix decrypts the message and forward $m$ ' to q. This scheme has been extended in [19, 20], 21] where several mixes are used to cope with the possibility of compromising the single mix. Another approach is to interpose an additional party (an anonymizer [22] between the sender and receiver to hide sender's identity from the receiver. Both the approaches are not viable in an ad hoc network for several reasons. First, they are based on the assumption that the information of mixes is known a priori and hence, the sender can select the mixes appropriate to the receiver. This assumption is impractical in an ad hoc network. Second, the mixes/anonymizers are entrusted with 
more responsibility and they can become single points of attack. Third, forwarding a packet through mixes/anonymizers results in much longer paths than the shortest paths possible, thus resulting in inefficient utilization of resources.

We address one routing attack that could easily happen in MANETs, the information disclosure problem. Specifically, we deal with the attack in which a malicious node may leak location information of other nodes.

\section{Protocol for Anonymous Routing}

In this section, we present a protocol to achieve absolute anonymity - the identities of the source and destinations are not known to any other node; after a connection is established, a node involved in the path does not even know its adjacent nodes in the path. Instead of containing source and destination information, packets moving along an anonymous connection contain only obscure information about next hop and previous hop.

\subsection{Notation and Definitions}

Public and Private Keys: We assume the presence of a Public Key Infrastructure. We denote the private and public keys of a node $i$ as $E_{i}$ and $D_{i}$. With $E(M, k)$ and $D(M, k)$ we denote the encryption and decryption of message $M$ with key $k$.

A Hash function, $H$, is assumed to be used globally; i.e., every node is aware of $H$ and uses $H$ to get the hash values.

Invisible Address $\left(I A_{i}\right)$ : We also define the invisible address $\left(I A_{i}\right)$ of a node $i$ for a packet with a flow identifier FID as constructed by encrypting the address along with FID first with the private key and then, with the public key of $i$ : $I A_{i}=E\left(E\left((i, F I D\right.\right.$, timestamp, RP $\left.\left.), E_{i}\right), D_{i}\right)$

$R P$ is the redundancy predicate. Node $\mathrm{N}$ to have its invisible address get verified, just presents $m=E\left((i, F I D\right.$, timestamp, $\left.R I P), E_{i}\right)$ to the verifier. For the message to be verified successfully the unencrypted message $D\left(m, D_{i}\right)$ must fulfill the redundancy predicate and $E\left(m, D_{i}\right)$ must be same as $I A_{i}$.

Routing Flow Table: Each node maintains a routing flow table (RFT), through which the node is able to forward a packet to the next node in the path. The information stored in each entry of the table is:

- Flow Identifier $(F I D)$ set to the unique request identifier present in the route request.

- Invisible Previous node Address (IPA) set to the invisible address of the node from which the route request is received.

- Invisible Next node Address $(I N A)$ set to the invisible address of the node from which the route received is received (if at all received).

- Timer $(T)$ initialized upon the reception of a non-duplicate route request to Th. The time $T h$ depends on the diameter of the network and could be set to the maximum Round Trip Time that could be possible in the network between any two nodes. The entry is deleted if a route reply is not received before the timer expires. 
We also assume that the network is very loosely synchronized. This assumption is just to prevent replay attacks.

\subsection{Route Requests}

Whenever a node $S$ wishes to communicate with a node $D$, it initiates the route discovery process. Route discovery allows any node in the ad hoc network to dynamically discover a route to any other node in the ad hoc network, whether directly reachable within wireless transmission range or reachable through one or more intermediate network hops through other nodes. A node initiating a route discovery broadcasts a route request, which may be received by those nodes within wireless transmission range of it.

The route request has the following fields:

- FID (Unique request identifier, also referred to as unique flow identifier) is set by the source by encrypting its address $(S)$, destination address $(D)$ and a locally maintained sequence number ( $S E Q$ ) with the public key of $S$. This is used to detect duplicate route requests received at an intermediate node: $F I D=E((S, D, S E Q), D S)$

- ESA (Encrypted Source Address) is constructed by encrypting source address, hash of FID, timestamp and the Redundancy Predicate $(R P)$ with the destination's public key. The hash of FID and the timestamp are to prevent replay attacks. $E S A=E((S, H(F I D)$, timestamp, $R P), D D)$

- EDA (Encrypted Destination Address) is constructed by encrypting destination address, hash of FID, timestamp and the Redundancy Predicate $(R P)$ with destination public key. $E D A=E((D, H(F I D)$, timestamp, $R P), D D)$

- ITA (Invisible Transmitter Address) is the invisible address of the node $i$ transmitting the route request. $I T A=E\left(E\left((i, F I D\right.\right.$, timestamp, $\left.\left.R P), E_{i}\right), D_{i}\right)$

Whenever a node $i$ that is not the destination receives a non-duplicate route request packet, it performs the following operations:

1. A new entry is added to the routing flow table with FID and IPA fields set to FID and ITA values of the route request packet.

2. The node checks if the route request is intended for it by decrypting the $E D A$ with its private key $E_{i}$ and if it is the case it proceeds to send a route reply (described below) and steps 3 and 4 are not executed.

3. The timer is initiated.

4. Invisible address is computed for the packet and the route request is retransmitted with its ITA set to the invisible address computed.

\subsection{Route Replies}

The destination after receiving the route request also adds a new entry to its $R F T$ in a similar manner as above. The destination also validates the source by decrypting $E S A$ with $E_{i}$. Then, the destination in order to establish a connection, constructs a route reply packet with the following fields: 
- FID is set to the FID of the route request.

- ESA (Encrypted Source Address) is constructed by encrypting D (destination of route request), hash of FID, timestamp and the Redundancy Predicate (RP) with the source's public key. The hash of FID and the timestamp are to prevent replay attacks. $E S A=E\left((D, H(F I D)\right.$, timestamp, $\left.R P), D_{S}\right)$

- ITA (Invisible Transmitter Address) is the invisible address of the node i transmitting the route request. IT $A=E\left(E\left((i, F I D\right.\right.$, timestamp, $\left.\left.R P), E_{i}\right), D_{i}\right)$

- IFA (Invisible Forwarder Address) is initially set to the ITA of the corresponding route request packet.

Whenever a node $i$ that is not the source, receives a route reply packet, it performs the following operations:

1. An entry corresponding to FID is searched for in the RFT. If no entry is found, the packet is dropped and all further steps are skipped.

2. The $I F A$ value is verified by checking for $R P$, its address, $F I D$ and the timestamp in $D\left(D\left(I F A, D_{i}\right), E_{i}\right)$. If the verification fails, the packet is dropped and all further steps are skipped.

3. The INA value of the entry corresponding to FID in RFT is set to ITA of the route reply and the timer of the corresponding entry is nullified.

4. The $I N A$ value of the route reply packet is set to the ITA value of the entry corresponding to FID and ITA value of the route reply is set to the invisible address of $i$. The route reply is then forwarded.

When the source receives the route reply, it can verify the destination address by decrypting the $E S A$ and $E D A$ fields in the route reply with its private key. After the verification, the source and destination can securely communicate with each other.

It should be noted that, no node in the network could make out the source or the destination of any packet/connection. Also, each node in the network does not even know the address of its neighboring node to which it is forwarding the packet. Thus, communication that is completely anonymous can be achieved. Also, apart from the overhead imposed due to the implementation of public key infrastructure, no extra overhead is imposed by our protocol. It should also be noted that, for each new connection, the route request is flooded over the whole network. To reduce the overhead, instead of pure flooding, protocols such as distance based flooding [11, gossip based flooding [12] or BSP [24] can be used.

\section{Enhanced Protocol for Anonymous Routing (PAR-E)}

With PAR, a malicious node can misuse the complete anonymity gained by transmitting fake routing requests. A misbehaving node, which drops the packets instead of retransmitting packets, can also go undetected. It is always a trade off between privacy and security. We propose a few enhancements to detect malicious and misbehaving nodes, albeit at the cost of complete anonymity. 
With the enhancements, a node will know the identity of any of its neighbors only if those two nodes lie on the same path of some connection. For instance, consider two neighboring nodes A and B. A will know the identity of B only if $\mathrm{A}$ and $\mathrm{B}$ lie in the path of some connection. If no such connection exists, then A does not know $B$ and vice versa.

We assume that all the nodes are aware of some symmetric key encryption algorithm and all nodes use the same symmetric key encryption algorithm. We denote the symmetric encryption and decryption processes of a message $M$ with key $k$ as $E_{s}(M, k)$ and $D_{s}(M, k)$.

\subsection{Enhancements}

Routing flow table: Five new fields are added to each entry of $R F T: 1) n$, a large prime chosen by the source. 2) $g$, a primitive mod n. 3) $x$, a large integer chosen for each entry by the node maintaining the RFT. 4)PPK (Previous node Partial Key) set to the partial key of the node from which the route request is received. 5) NPK (Next node Partial Key) set to the partial key of the node from which the route reply is received (if at all received).

Route request: Three new fields are added to the route request packet: 1) $n$, a large prime chosen by the source. 2) $g$, a primitive mod n. 3)TPK, Transmitter partial key, computed and set by the transmitter as $T P K=g^{x} \bmod n$

Route reply: Five new fields are added to the route reply packet 1) $n$, large prime chosen by the source of route request. 2) $g$, a primitive mod $n$ and chosen by $S .3) N P K$, next node partial key set by the transmitter. 4) $T V$, Transmitter Verifier, set to the cipher text obtained by encrypting the transmitter's address and its signature with $S K$ as key. $S K$ is computed using $P P K$ and $x$ as $S K=$ $\left(P P K^{x} \bmod n\right)$, and $T V=E s\left(\left(T r \_a d d r e s s\right.\right.$, signature $\left.\left.), S K\right) 5\right) T V$ ', Previous Transmitter Verifier, set to the cipher text obtained by encrypting the transmitter's address and its signature with $S K^{\prime}$ as key. $S K^{\prime}$ is computed using $N P K$ and $x$ as $S K^{\prime}=\left(N P K^{x} \bmod n\right)$ and $T V^{\prime}=E s\left(\left(T r_{-}\right.\right.$address, signature $\left.), S K^{\prime}\right)$

Initially, the source node $S$ chooses a large prime, $n$ and $g$, such that $g$ is primitive $\bmod n$ and initializes the corresponding fields in the route request to these. Any other node, that is not the destination, upon reception of a route request, apart from steps 1 - 4 (Section 4.2), performs an additional step 3a: The node chooses a large integer $x$ and sets the field $x$ in the newly created entry to that integer. Set the $P P K$ field in the $R F T$ entry to $T P K$ of the route request and reset the $T P K$ entry of route request to $g^{x} \bmod n$.

The destination $D$ upon receiving the route request creates a new entry in its RFT and sets its fields to the corresponding fields of route request. It then generates a large integer $\mathrm{x}$ and computes the shared key according to DiffieHellman key Exchange algorithm as : $S K=T P K^{x} \bmod n$.

Then, $D$ constructs the route reply as explained in Section 4.3 with the new fields set in the following way: $n$, set to the large prime present in the route request. $g$, set to $g$ present in the route request. $N P K$, next node partial key, set to $\left(g^{x} \bmod n\right), x$ being a large integer, chosen by $D . S K$, shared key, is calculated as $S K=\left(T P K^{x} \bmod n\right), T P K$ being transmitter partial key obtained from 
the route request. $T V$, Transmitter Verifier, set to the cipher text obtained by encrypting the transmitter's address and its signature with $S K$ as key. $T V=$ $E_{s}((D$, signature $), S K)$. TV', Transmitter Verifier.

A node $i$, that is not the source, upon reception of a route reply, apart from steps 1 - 4 (Section 4.3), performs an additional step 3a: The node computes the shared keys, $S K$ and $S K^{\prime}$ as $\left(N P K^{x} \bmod n\right) \operatorname{and}\left(P P K^{x} \bmod n\right), x$ being the value in the $R F T$ entry corresponding to FID. Using $S K, T V$ is verified by decrypting it with the $S K$. Upon verification, it sets $N P K$ field of route reply to $\left(g^{x} \bmod n\right), x$ taken from $R F T$ entry. Using TPK from the RFT entry, the node calculates $S K=\left(T P K^{x} \bmod n\right)$ and resets the Transmitter Verifier in the route reply to $T V=E_{s}((i$, signature $), S K)$. It then retransmits the route reply.

Also, after retransmitting route reply, then node i overhears the route reply its neighbor retransmits for $T V^{\prime}$ and verifies the signature. In case of a node next to source, the source explicitly transmits TV' for the node to verify its identity. When the source receives the route reply, it verifies the destination address by decrypting the ESA and EDA fields in the route reply with its private key. Thus, secure communication channel between the source and the destination is established. It should be observed that each node in the path established knows nothing more than the identities of its neighboring nodes in the path established. The identities of even other neighboring nodes are revealed. As each node knows the identity of its neighboring nodes in the paths established, Intrusion Detection Systems such as [13, 14, 15] can be implemented successfully to detect malicious and misbehaving routers.

\section{Conclusion}

In this paper we presented protocols for achieving anonymous routing in mobile ad hoc networks and thus, prevent location disclosure attacks. The protocol for Anonymous Routing (PAR) guarantees absolute anonymity, which itself might cause problems as it would become hard to identify malicious and misbehaving nodes. PAR-Enhanced trades off some anonymity to enable detection of malicious and misbehaving nodes.

\section{References}

1. Vesa Karpijoki: Security in Ad hoc Networks. In Proceedings of the Helsinki University of Technology, Seminars on Network Security, Helsinki, Finland, 2000.

2. L. Zhou and Z. J. Haas: Securing ad hoc networks. IEEE Network Magazine, 13(6):24-30, November/December 1999.

3. Janne Lundberg: Routing Security in Ad Hoc Networks. http://citeseer.nj.nec.com/400961.html

4. F. Stajano and R.J. Anderson: The Resurrecting Duckling: Security Issues in AdHoc Wireless Networks. Proc. Seventh Security Protocols Workshop, Lecture Notes in Computer Science 1796, Springer-Verlag, Berlin, 2000, pp. 172-182.

5. H. Deng, W. Li, D. Agrawal: Routing Security in Wireless Ad Hoc Networks. IEEE Communications Magazine, Oct. 2002, pp. 70-75. 
6. Lakshmi Venkatraman and Dharma P. Agrawal: Startegies for Enhancing Routing Security in Protocols for Mobile Ad hoc Networks. JPDC Special Issue on Mobile Ad Hoc Networking and Computing, accepted for publication.

7. C. R. Davis: IPSec: Securing VPNs. McGraw-Hill, New York, NY, USA, 2000.

8. L. Zhou and J. Haas: Securing ad hoc networks. IEEE Network, 13(6): 24-30, 1999.

9. J. P. HuBaux, L. Buttyan, and S. Capkun: The quest for security in mobile ad hoc networks. In Proc. ACM MobiHoc, October 2001.

10. S. Yi, P. Naldurg, and R. Kravets: Security-aware ad hoc routing for wireless networks. Technical Report UIUCDCS-R-2001- 2241, UILU-ENG-2001-1748, University of Illinois at Urbana-Champaign, August 2001

11. S. Y. Ni et al: The Broadcast Storm Problem in a Mobile Ad Hoc Network. ACM MOBICOM, pp. 151-162, Aug' 1999.

12. Haas, Halpern, Li: Gossip based Ad Hoc Routing. In IEEE INFOCOM, June 2002.

13. Oleg Kachirski, Ratan Guha: Intrusion Detection Using Mobile Agents in Wireless Ad Hoc Networks, IEEE Workshop on Knowledge Media Networking.

14. R. Ramanujan, S. Kudige, T. Nguyen, S. Takkella, and F. Adelstein: IntrusionResistant Ad Hoc Wireless Networks, Proceedings of MILCOM 2002, Oct. 2002.

15. Yongguang Zhang and Wenke Lee: Intrusion Detection in Wireless Ad-Hoc Networks, Proceedings of The Sixth International Conference on Mobile Computing and Networking, Boston, MA, August 2000

16. Pfitzmann, A. and Waidner, M: Networks without user observability. Computer Security, 6/2 (1987) 158-166.

17. M. K. Reiter and A. D. Rubin: Crowds: Anonymity for Web Transactions. ACM Transactions on Information and System Security, pp. 66-92, 1(1), 1998.

18. D. Chaum: Untraceable electronic mail, return addresses, and digital pseudonyms. Communication of the ACM, vol. 24, no. 2, pp. 84-88, 1981.

19. A. Pfitzmann, B. Pfitzmann, and M. Waidner: ISDN-MIXes - Untraceable communication with very small bandwidth overhead. In Proc. Kommunikation in verteilten Systemen, pp. 451-463, 1991.

20. C. Rackoff and D. Simon: Cryptographic defense against traffic analysis. In Proc. of the 25th Annu. ACM Symp. on the Theory of Computing, pp. 672- 681, 1993.

21. P. F. Syverson, D. M. Goldsclag, and M. G. Reed: Anonymous connections and onion routing. In Proc. of IEEE Symposium on Security and Privacy, pp. 44-54, 1997

22. Anonymizer - Online Privacy and Security. www.anonymizer.com

23. D. Niculescu and B. Nath: Ad Hoc Positioning System (APS) using AoA, INFOCOM. 03, San Francisco, CA, 2003.

24. Arjan Durresi, Vamsi Paruchuri, Sitharama Iyengar, Rajgopal Kannan: Optimized Broadcast Protocol for Sensor Networks, IEEE Transactions on Computers, Vol. 54, No. 8, August, 2005, pp. 1013-1024. 